\title{
Endophytic bacteria isolated from Phaseolus vulgaris produce phytases with potential for biotechnology application
}

\section{Leonardo Emanuel de Oliveira Costa, Thamy Lívia Ribeiro Corrêa, Janaina Aparecida Teixeira, Elza Fernandes de Araújo and Marisa Vieira de Queiroz*}

Departamento de Microbiologia/BIOAGRO. Universidade Federal de Viçosa. Av. P. H. Rolfs, S/№. Viçosa-MG, Brazil (CEP 36570-000).*E-mail: mvqueiro@ufv.br.

\begin{abstract}
Currently, endophytic microorganisms have become a good source of different enzymes and others metabolites of industrial interest. Among a huge spectral of molecules, enzymes as phytases have been emphasized by the ability to hydrolyze the phytic acid that represents the largest storage form of inorganic phosphorus in cereals, which are the staple diet of monogastric animals such as swine and poultry. Moreover, phytic acid acts as an antinutrient by chelating divalent metal ions, and it is interesting provide phytase as an animal feed supplement for those monogastric animals. In the current study, 158 endophytic bacteria isolated from the leaves of three cultivars of Phaseolus vulgaris were assessed for the ability to produce phytase. Among them, four isolates belonging to the Pseudomonas, Stenotrophomonas, Microbacterium and Rhodococcus genera were highlighted, due their phytase production. The phytase produced by Microbacterium foliorum BAC1157 exhibited activity at $70^{\circ} \mathrm{C}$ and stability in the presence of divalent cations, indicating that this phytase has a promising use in the animal feed industry. To the authors' knowledge, this is the first report on phytase production by bacteria of the Microbacterium genera.
\end{abstract}

Keywords: Endophytic bacteria; Microbacterium; Bean; Phytase activity.

\footnotetext{
.
}

Received

October 1, 2018

Accepted

November 23, 2018

Released

December 31, 2018

Full Text Article

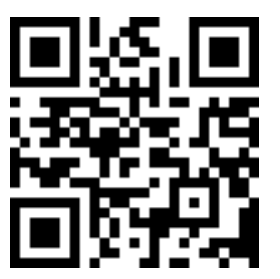

ORCID

Do 0000-0003-4375-9298

Leonardo Emanuel de Oliveira Costa

(D) 0000-0003-4270-6530

Thamy Lívia Ribeiro Corrêa

(D) 0000-0002-3839-0943

Janaina Aparecida

Teixeira

(D) 0000-0002-5177-5776

Elza Fernandes de

Araújo

(D) 0000-0002-2280-4793

Marisa Vieira de

Queiroz 


\section{Introduction}

Endophytes are microorganisms that can be isolated from surfacedisinfested healthy plant tissue and do not harm the host plant (Hallmann et al., 1997). These microorganisms are considered potential sources of compounds such as antibiotics, antioxidants, anticancer drugs and enzymes (Strobel and Daisy, 2003). Phytase activity were detected in the endophytic bacteria belonging the genera Bacillus, Streptomyces, Acinetobacter, and Rhizobium isolated from the seeds and roots of Phaseolus vulgaris (common bean) (López-López et al., 2010). The phytases, or myo-inositol hexakisphosphate phosphohydrolases (E.C. 3.1.3.8; 3.1.3.26; 3.1.3.72), are derived from various sources, including animals, plants and microorganisms (Konietzny and Greiner, 2002; Vohra and Satyanarayana, 2003). These enzymes are notable for being able to hydrolyze phytate making available the inorganic phosphorus (Pi) and phosphorylated myo-inositols (Ariza et al., 2013). Phytic acid (myo-inositol hexakisphosphate) and the mixture of the cationic salts of phytic acid, which is commonly known as phytate, are organic phosphorus compounds that are widespread in nature. Approximately $60 \%$ to $90 \%$ of the $\mathrm{Pi}$ in vegetables, oilseeds and cereals is stored in the form of phytic acid (Coelho et al., 2002; Rao et al., 2009).

Monogastric animals such as swine and poultry are unable to degrade the phytic acid in food (Schroder et al., 1996). Therefore, the diet of these animals should be supplemented with $\mathrm{Pi}$ to meet their nutritional needs. However, approximately $70 \%$ of the $\mathrm{Pi}$ supplemented in diets is excreted into the environment, triggering the eutrophication of bodies of water near farm areas (Turner and Haygarth, 2000). Furthermore, phytic acid acts as an antinutritional factor by chelating divalent cations (such as $\mathrm{Zn}^{2+}, \mathrm{Fe}^{2+/ 3+}$, $\mathrm{Ca}^{2+}, \mathrm{Mg}^{2+}, \mathrm{Mn}^{2+}$ and $\mathrm{Cu}^{2+}$, proteins, starch and lipids, thus preventing their absorption throughout the gastrointestinal tract (Urbano et al., 2000). Thus, the importance to add feed enzymes on animal diet is that it can have an effect on the microbiome of the gastrointestinal tract promoting gut absorption of Pi and other cations for the animals (Kiarie et al. 2013; Tortola et al., 2013).

Most of the microbial phytases described in the literature are acidic phytases, with an optimal $\mathrm{pH}$ and temperature range of 4.0 to 5.5 and $45^{\circ} \mathrm{C}$ to $60{ }^{\circ} \mathrm{C}$, respectively (Lei et al., 2003; Mullaney and Ullah, 2003; Patki et al., 2015). The optimal temperature for most microbial phytases is approximately $50{ }^{\circ} \mathrm{C}$ (Simon and Igbasan, 2002); however, some microbial phytases have exhibited optimal temperatures above $60{ }^{\circ} \mathrm{C}$ (Berka et al., 1998; Matsui et al., 2000; Zamudio et al., 2001). These enzymes can dephosphorylate phytate into several position isomers of myoinositol pentakis-, tetrakis-, tris-, bis- and monophosphates (Greiner et al., 2001). Several myo-inositol isomers show pharmacological antitumor and antiangiogenic (Maffucci et al., 2005), and anti-inflammatory (Claxson et al., 1990) effects, in addition to preventing diabetes complications (Carrington et al., 1993). For these and other reasons, phytases have tremendous potential in biotechnology, especially human and animal nutrition and bioremediation.

The global market for feed enzyme has growing in the next years; it has valued at \$899.19 Million in 2014 and is estimated to reach $\$ 1,371.03$ Million by 2020 according to market research firm - MarketsandMarkets (http://www.marketsandmarkets.com). Among the major enzymes applied, phytases correspond for $60 \%$ to $80 \%$ of the market for animal nutrition (Corrêa et al., 2015). Thus, endophytic microorganisms that are promising sources of phytases have been evaluated. Accordingly, the aim of this study was to assess the phytases produced by the 
endophytic bacteria isolated from three varieties of the common bean, Phaseolus vulgaris (Costa et al., 2012), and to characterize the enzymes from these promising bacterial isolates.

\section{Matherials and methods}

One hundred fifty-eight strains of endophytic bacteria were isolated from the leaves of the Talismã, Ouro Negro and Vermelhinho cultivars of the common bean (Costa et al., 2012) and used for screening phytase production. The bacterial isolates were activated in $10 \mathrm{~mL}$ of $10 \%$ TSA culture media (Araujo et al., 2002) at $28{ }^{\circ} \mathrm{C}$ and $150 \mathrm{rpm}$ for 48 to $72 \mathrm{~h}$. Each bacterial isolate was inoculated into the phytase screening media plates described by Kim et al. (2003) Phytase production was assessed by the formation of a hydrolysis halo around the colonies 48 and $96 \mathrm{~h}$ after incubation. Bacterial isolates showing larger hydrolysis halos were selected for further testing. The strains selected were activated in TSA media as previously described to quantify phytase activity. An aliquot of the cell culture was used to inoculate $50 \mathrm{~mL}$ of phytase screening media (PSM), which consisted of $1.5 \mathrm{~g} \mathrm{~L}^{-1}$ glucose, $0.5 \mathrm{~g} \mathrm{~L}^{-1} \mathrm{NH}_{4} \mathrm{NO}_{3}, 0.05 \mathrm{~g} \mathrm{~L}^{-1} \mathrm{KCl}$, $0.05 \mathrm{~g} \quad \mathrm{~L}^{-1} \mathrm{MgSO}_{4} .7 \mathrm{H}_{2} \mathrm{O}, \quad 0.001 \mathrm{~g} \mathrm{~L}^{-1}$ $\mathrm{FeSO}_{4} .7 \mathrm{H}_{2} \mathrm{O}, 0.001 \mathrm{~g} \mathrm{~L}^{-1} \mathrm{MnSO}_{4} .7 \mathrm{H}_{2} \mathrm{O}$ and $5.00 \mathrm{~g} \mathrm{~L}^{-1}$ phytic acid calcium salt $\left(\mathrm{C}_{6} \mathrm{H}_{16} \mathrm{CaO}_{24} \mathrm{P}_{6}\right.$ - $\mathrm{P} 9539-$ Sigma $\left.^{\circledR}\right)$ at a $\mathrm{pH}$ of 5.5. The cultures were maintained at 28 ${ }^{\circ} \mathrm{C}$ and $150 \mathrm{rpm}$ for $48 \mathrm{~h}$. After this period, the broth was centrifuged at $3,000 \mathrm{~g}$ for $5 \mathrm{~min}$, and the supernatant was used to determine the enzymatic activity.

In order to characterize the enzyme produced by the endophytic isolates, the phytase activity was determined by incubating $50 \mu \mathrm{L}$ of supernatant in $350 \mu \mathrm{L}$ of $0.1 \mathrm{M}$ sodium acetate buffer, pH 5.0, with $2.5 \mathrm{mM}$ phytic acid sodium salt $\left(\mathrm{C}_{6} \mathrm{H}_{18} \mathrm{O}_{24} \mathrm{P}_{6} \mathrm{xNa}^{+}\right.$ $\mathrm{yH}_{2} \mathrm{O}$ - $\mathrm{P} 0109-$ Sigma $^{\circledR}$ ) at $50{ }^{\circ} \mathrm{C}$ for 15 min. The Pi released was quantified using the method described by Heinonen and
Lahti (1981). A standard curve with several concentrations of $\mathrm{KH}_{2} \mathrm{PO}_{4}$ was used to calculate the enzymatic activity. One unit of phytase activity was defined as the amount of enzyme required to release $1 \mu \mathrm{mol}$ of phosphate per minute under the assay conditions. The effect of temperature on phytase activity was measured by incubating the culture filtrate with the substrate at temperatures ranging from $20^{\circ} \mathrm{C}$ to $80^{\circ} \mathrm{C}$ for $15 \mathrm{~min}$. The effect of $\mathrm{pH}$ on enzyme activity was assessed by incubating the culture supernatant and the substrate in the following $(0.1 \mathrm{M})$ buffer solutions: glycine- $\mathrm{HCl}(\mathrm{pH} 1.0-3.5)$, sodium acetate (pH 4.0-6.0), Tris-HCl (pH 6.5-8.5) and glycine- $\mathrm{NaOH} \quad(\mathrm{pH}$ 9.0-12.0). The enzyme-substrate specificity was determined by replacing the phytic acid sodium salt with $2.5 \mathrm{mM}$ ATP, ADP, pyrophosphate or $\beta$-glycerophosphate. The effect of divalent cations on phytase activity was determined by incubating the supernatant of each bacterial isolate with $1 \mathrm{mM}, 5 \mathrm{mM}, 10 \mathrm{mM}$ or $20 \mathrm{mM}$ $\mathrm{CaCl}_{2}, \mathrm{CdSO}_{4}, \mathrm{CuSO}_{4}, \mathrm{FeSO}_{4}, \mathrm{KCl}, \mathrm{MgCl}_{2}$, $\mathrm{MnCl}_{2}$ or $\mathrm{ZnSO}_{4}$ for $1 \mathrm{~h}$. The $500 \mathrm{mM}$ stock solution of each cation was prepared as described in Tang et al. (2006). The untreated enzyme activity was set to $100 \%$ in all of the assays. The data were analyzed using analysis of variance and Tukey's test at 5\% probability using SAEG software, version 9.1, Universidade Federal de Viçosa.

The 158 bacterial isolates were inoculated in solid media with calcium phytate to qualitatively assess phytase production. Among them, 45 (28.48\%) exhibited a hydrolysis halo around the colonies $48 \mathrm{~h}$ and $96 \mathrm{~h}$ after inoculation (Table 1). The most promising isolates with hydrolysis halos were Microbacterium foliorum BAC1157, Pseudomonas aeruginosa BAC3115, Rhodococcus erythropolis BAC2162 and Stenotrophomonas maltophilia BAC2135, with $15.5 \mathrm{~cm}$ (48 h), $22.0 \mathrm{~cm}(96 \mathrm{~h}), 15.0$ $\mathrm{cm}(48 \mathrm{~h})$ and $22.0 \mathrm{~cm}(96 \mathrm{~h})$ hydrolysis halos, respectively. The bacterial isolates were subsequently cultured in liquid 
induction media to quantify phytase production (Figure 1). Of these cultures, $R$. erythropolis BAC2162 and $S$. maltophilia BAC2135 showed the highest phytase activities $(0.659$ and 0.625 $\mathrm{U} \mathrm{mL}^{-1}$, respectively), while $M$. foliorum BAC1157 showed the lowest activity (0.550 $\left.\mathrm{U} \mathrm{mL}^{-1}\right)$.

Table 1. The in vitro phytase production assay from the 158 endophytic isolates of the $P$. vulgaris leaf and hydrolysis halo size of the 45 isolates positive for phytase production.

\begin{tabular}{|c|c|c|c|c|}
\hline \multirow[t]{2}{*}{ Isolate } & \multirow{2}{*}{$\begin{array}{l}\text { Number of } \\
\text { access }\end{array}$} & \multirow{2}{*}{$\begin{array}{l}\text { Phytase } \\
\text { production }\end{array}$} & \multicolumn{2}{|c|}{$\begin{array}{l}\text { Halo size } \\
\text { (cm) }\end{array}$} \\
\hline & & & $48 h$ & $96 \mathrm{~h}$ \\
\hline Bacillus thuringiensis strain BAC1001 & HM355592 & - & 0 & 0 \\
\hline Micrococcus luteus strain BAC1002 & HM355593 & - & 0 & 0 \\
\hline Microbacterium testaceum strain BAC1003 & HM355594 & - & 0 & 0 \\
\hline Micrococcus luteus strain BAC1004 & HM355595 & + & 14.5 & 17 \\
\hline Micrococcus luteus strain BAC1005 & HM355596 & - & 0 & 0 \\
\hline Microbacterium testaceum strain BAC1006 & HM355597 & + & 12.5 & 14.5 \\
\hline Enterobacter asburiae strain BAC1007 & HM355598 & + & 13 & 15 \\
\hline Microbacterium testaceum strain BAC 1008 & HM355599 & - & 0 & 0 \\
\hline Microbacterium testaceum strain BAC1009 & HM355600 & + & 11 & 15 \\
\hline Enterobacter hormaechei strain BAC1010 & HM355601 & + & 12 & 15 \\
\hline Rhizobium larrymoorei strain BAC1011 & HM355602 & - & 0 & 0 \\
\hline Stenotrophomonas maltophilia strain BAC1012 & HM355603 & + & 14 & 17,5 \\
\hline Microbacterium testaceum strain BAC1013 & HM355604 & + & 14 & 17 \\
\hline Microbacterium testaceum strain BAC1014 & HM355605 & + & 12 & 15 \\
\hline Bacillus niacini strain BAC1015 & HM355606 & - & 0 & 0 \\
\hline Staphylococcus epidermidis strain BAC1016 & HM355607 & - & 0 & 0 \\
\hline Bacillus thuringiensis strain BAC1017 & HM355608 & + & 12.5 & 15.5 \\
\hline Microbacterium testaceum strain BAC1018 & HM355609 & - & 0 & 0 \\
\hline Microbacterium testaceum strain BAC1019 & HM355610 & - & 0 & 0 \\
\hline Microbacterium testaceum strain BAC1020 & HM355611 & - & 0 & 0 \\
\hline Rhizobium larrymoorei strain BAC2021 & HM355612 & + & 12 & 16 \\
\hline Microbacterium testaceum strain BAC2022 & HM355613 & - & 0 & 0 \\
\hline Methylobacterium populi strain BAC2023 & HM355614 & - & 0 & 0 \\
\hline Stenotrophomonas maltophilia strain BAC2024 & HM355615 & - & 0 & 0 \\
\hline Rhizobium larrymoorei strain BAC2025 & HM355616 & - & 0 & 0 \\
\hline Staphylococcus epidermidis strain BAC2026 & HM355617 & + & 9.5 & 16 \\
\hline Brevibacillus agri strain BAC2027 & HM355618 & + & 13 & 16 \\
\hline Methylobacterium populi strain BAC2028 & HM355619 & + & 12.5 & 17.5 \\
\hline Microbacterium testaceum strain BAC2029 & HM355620 & - & 0 & 0 \\
\hline Staphylococcus warneri strain BAC2030 & HM355621 & + & 10 & 16 \\
\hline Stenotrophomonas maltophilia strain BAC2031 & HM355622 & - & 0 & 0 \\
\hline Staphylococcus warneri strain BAC2032 & HM355623 & - & 0 & 0 \\
\hline Methylobacterium populi strain BAC2033 & HM355624 & + & 7 & 12.5 \\
\hline Staphylococcus epidermidis strain BAC2034 & HM355625 & - & 0 & 0 \\
\hline Staphylococcus epidermidis strain BAC2035 & HM355626 & - & 0 & 0 \\
\hline Microbacterium testaceum strain BAC2036 & HM355627 & + & 0 & 4 \\
\hline Sphingomonas dokdonensis strain BAC2037 & HM355628 & - & 0 & 0 \\
\hline Sporosarcina aquimarina strain BAC2038 & HM355629 & - & 0 & 0 \\
\hline Staphylococcus caprae strain BAC2039 & HM355630 & - & 0 & 0 \\
\hline Methylobacterium populi strain BAC2040 & HM355631 & - & 0 & 0 \\
\hline Staphylococcus epidermidis strain BAC3041 & HM355632 & - & 0 & 0 \\
\hline
\end{tabular}


Table 1. Continued.

\begin{tabular}{|c|c|c|c|c|}
\hline \multirow[t]{2}{*}{ Isolate } & \multirow{2}{*}{$\begin{array}{l}\text { Number of } \\
\text { access }\end{array}$} & \multirow{2}{*}{$\begin{array}{c}\text { Phytase } \\
\text { production }\end{array}$} & \multicolumn{2}{|c|}{$\begin{array}{l}\text { Halo size } \\
\text { (cm) }\end{array}$} \\
\hline & & & $48 \mathrm{~h}$ & $96 \mathrm{~h}$ \\
\hline Bacillus thuringiensis strain BAC 3042 & HM355633 & - & 0 & 0 \\
\hline Microbacterium foliorum strain BAC 3043 & HM355634 & + & 12 & 15.5 \\
\hline Bacillus bataviensis strain BAC3044 & HM355635 & - & 0 & 0 \\
\hline Sphingobacterium multivorum strain BAC3045 & HM355636 & + & 11.5 & 14.5 \\
\hline Staphylococcus epidermidis strain BAC3046 & HM355637 & - & 0 & 0 \\
\hline Microbacterium testaceum strain BAC 3047 & НM355638 & - & 0 & 0 \\
\hline Bacillus amyloliquefaciens strain BAC3048 & НM355639 & - & 0 & 0 \\
\hline Staphylococcus kloosii strain BAC3049 & HМ355640 & - & 0 & 0 \\
\hline Microbacterium phyllosphaerae strain BAC3050 & HM355641 & - & 0 & 0 \\
\hline Methylobacterium populi strain BAC3051 & HM355642 & - & 0 & 0 \\
\hline Stenotrophomonas maltophilia strain BAC3052 & HM355643 & - & 0 & 0 \\
\hline Bacillus bataviensis strain BAC 3053 & НM355644 & - & 0 & 0 \\
\hline Methylobacterium populi strain BAC 3054 & HM355645 & - & 0 & 0 \\
\hline Staphylococcus warneri strain BAC3055 & HM355646 & - & 0 & 0 \\
\hline Sporosarcina sp. strain BAC3056 & HM355647 & - & 0 & 0 \\
\hline Acinetobacter sp. strain BAC 3057 & HМ355648 & - & 0 & 0 \\
\hline Acinetobacter radioresistens strain BAC3058 & НM355649 & + & 14.5 & 16 \\
\hline Lysinibacillus sphaericus strain BAC3059 & НM355650 & - & 0 & 0 \\
\hline Microbacterium testaceum strain BAC1061 & HM355651 & - & 0 & 0 \\
\hline Staphylococcus epidermidis strain BAC1062 & HM355652 & - & 0 & 0 \\
\hline Bacillus thuringiensis strain BAC1063 & HМ355653 & - & 0 & 0 \\
\hline Methylobacterium populi strain BAC1064 & HM355654 & + & 14 & 18.5 \\
\hline Microbacterium testaceum strain BAC1065 & HM355655 & - & 0 & 0 \\
\hline Microbacterium testaceum strain BAC1066 & HM355656 & - & 0 & 0 \\
\hline Microbacterium foliorum strain BAC1067 & HM355657 & - & 0 & 0 \\
\hline Methylobacterium populi strain BAC1068 & HМ355658 & - & 0 & 0 \\
\hline Methylobacterium populi strain BAC1069 & HM355659 & - & 0 & 0 \\
\hline Brevundimonas vesicularis strain BAC1070 & HM355660 & - & 0 & 0 \\
\hline Staphylococcus epidermidis strain BAC2071 & HM355661 & - & 0 & 0 \\
\hline Frigoribacterium faeni strain BAC2072 & HM355662 & - & 0 & 0 \\
\hline Sphingomonas sanguinis strain BAC2073 & HM355663 & - & 0 & 0 \\
\hline Staphylococcus warneri strain BAC2074 & HM355664 & - & 0 & 0 \\
\hline Frigoribacterium faeni strain BAC2075 & HM355665 & - & 0 & 0 \\
\hline Rhizobium larrymoorei strain BAC2076 & HM355666 & - & 0 & 0 \\
\hline Staphylococcus epidermidis strain BAC2077 & HM355667 & - & 0 & 0 \\
\hline Bacillus subtilis strain BAC2078 & HМ355668 & - & 0 & 0 \\
\hline Microbacterium testaceum strain BAC2079 & HM355669 & - & 0 & 0 \\
\hline Microbacterium testaceum strain BAC2080 & HM355670 & - & 0 & 0 \\
\hline Brevibacillus agri strain BAC3081 & HM355671 & - & 0 & 0 \\
\hline Methylobacterium populi strain BAC 3082 & НM355672 & - & 0 & 0 \\
\hline Methylobacterium populi strain BAC 3083 & HМ355673 & - & 0 & 0 \\
\hline Paenibacillus cineris strain BAC3084 & HМ355674 & - & 0 & 0 \\
\hline Staphylococcus epidermidis strain BAC3085 & HM355675 & - & 0 & 0 \\
\hline Microbacterium foliorum strain BAC 3087 & HМ355676 & - & 0 & 0 \\
\hline Methylobacterium populi strain BAC 3088 & НM355677 & - & 0 & 0 \\
\hline Staphylococcus warneri strain BAC3089 & HМ355678 & - & 0 & 0 \\
\hline Methylobacterium populi strain BAC 3090 & HM355679 & - & 0 & 0 \\
\hline Paenibacillus cineris strain BAC1091 & HM355680 & + & 0 & 8 \\
\hline Microbacterium testaceum strain BAC1092 & HM355681 & - & 0 & 0 \\
\hline Microbacterium testaceum strain BAC1093 & HM355682 & - & 0 & 0 \\
\hline Microbacterium phyllosphaerae strain BAC1094 & HM355683 & - & 0 & 0 \\
\hline
\end{tabular}


Table 1. Continued.

\begin{tabular}{|c|c|c|c|c|}
\hline \multirow[t]{2}{*}{ Isolate } & \multirow{2}{*}{$\begin{array}{l}\text { Number of } \\
\text { access }\end{array}$} & \multirow{2}{*}{$\begin{array}{l}\text { Phytase } \\
\text { production }\end{array}$} & \multicolumn{2}{|c|}{$\begin{array}{l}\text { Halo size } \\
\text { (cm) }\end{array}$} \\
\hline & & & $48 \mathrm{~h}$ & $96 \mathrm{~h}$ \\
\hline Micrococcus luteus strain BAC1095 & HM355684 & - & 0 & 0 \\
\hline Bacillus muralis strain BAC1096 & HM355685 & - & 0 & 0 \\
\hline Microbacterium sp. strain BAC1097 & HM355686 & - & 0 & 0 \\
\hline Kocuria palustris strain BAC1098 & HM355687 & - & 0 & 0 \\
\hline Microbacterium testaceum strain BAC1099 & HM355688 & - & 0 & 0 \\
\hline Microbacterium testaceum strain BAC1100 & HM355689 & - & 0 & 0 \\
\hline Staphylococcus saprophyticus strain BAC2101 & HM355690 & - & 0 & 0 \\
\hline Staphylococcus warneri strain BAC2102 & HM355691 & - & 0 & 0 \\
\hline Methylobacterium populi strain BAC2103 & HM355692 & - & 0 & 0 \\
\hline Delftia tsuruhatensis strain BAC2104 & HM355693 & - & 0 & 0 \\
\hline Methylobacterium populi strain BAC2105 & HM355694 & - & 0 & 0 \\
\hline Bacillus sp. strain BAC2106 & HM355695 & - & 0 & 0 \\
\hline Staphylococcus warneri strain BAC2107 & HM355696 & - & 0 & 0 \\
\hline Methylobacterium populi strain BAC2108 & HM355697 & - & 0 & 0 \\
\hline Staphylococcus warneri strain BAC2109 & HM355698 & - & 0 & 0 \\
\hline Frigoribacterium faeni strain BAC2110 & HM355699 & - & 0 & 0 \\
\hline Pseudomonas aeruginosa strain BAC3111 & HM355700 & + & 13 & 17.5 \\
\hline Staphylococcus warneri strain BAC3112 & HM355701 & + & 12.5 & 14.5 \\
\hline Methylobacterium populi strain BAC3113 & HM355702 & - & 0 & 0 \\
\hline Dietzia cinnamea strain BAC3114 & HM355703 & - & 0 & 0 \\
\hline Pseudomonas aeruginosa strain BAC 3115 & HM355704 & + & $15.5 \dagger$ & 19.5 \\
\hline Staphylococcus warneri strain BAC3116 & HM355705 & - & 0 & 0 \\
\hline Agromyces mediolanus strain BAC 3117 & HM355706 & - & 0 & 0 \\
\hline Agromyces mediolanus strain BAC 3118 & HM355707 & - & 0 & 0 \\
\hline Staphylococcus warneri strain BAC3119 & HM355708 & - & 0 & 0 \\
\hline Stenotrophomonas sp. strain BAC3120 & HM355709 & - & 0 & 0 \\
\hline Stenotrophomonas maltophilia strain BAC 3121 & HM355710 & - & 0 & 0 \\
\hline Stenotrophomonas maltophilia strain BAC 3122 & HM355711 & - & 0 & 0 \\
\hline Stenotrophomonas maltophilia strain BAC 3123 & HM355712 & - & 0 & 0 \\
\hline Agromyces mediolanus strain BAC 3124 & HM355713 & - & 0 & 0 \\
\hline Stenotrophomonas maltophilia strain BAC3125 & HM355714 & - & 0 & 0 \\
\hline Microbacterium testaceum strain BAC2126 & HM355715 & + & 14.5 & 20.5 \\
\hline Stenotrophomonas maltophilia strain BAC2127 & HM355716 & - & 0 & 0 \\
\hline Stenotrophomonas maltophilia strain BAC2128 & HM355717 & + & 12.5 & 16 \\
\hline Agromyces mediolanus strain BAC2129 & HM355718 & - & 0 & 0 \\
\hline Agromyces sp. strain BAC2130 & HM355719 & - & 0 & 0 \\
\hline Staphylococcus epidermidis strain BAC2131 & HM355720 & - & 0 & 0 \\
\hline Methylobacterium populi strain BAC2132 & HM355721 & - & 0 & 0 \\
\hline Paenibacillus lautus strain BAC2133 & HM355722 & - & 0 & 0 \\
\hline Stenotrophomonas maltophilia strain BAC2134 & HM355723 & + & 10.5 & 15 \\
\hline Stenotrophomonas maltophilia strain BAC2135 & HM355724 & + & 14.5 & $22.0 \dagger$ \\
\hline Stenotrophomonas maltophilia strain BAC1136 & HM355725 & + & 10 & 15 \\
\hline Pseudomonas aeruginosa strain BAC1137 & HM355726 & + & 13 & 16.5 \\
\hline Enterobacter asburiae strain BAC1138 & HM355727 & + & 12.5 & 15 \\
\hline Enterobacter hormaechei strain BAC1139 & HM355728 & + & 13.5 & 16.5 \\
\hline Bacillus thuringiensis strain BAC1140 & HM355729 & + & 13.5 & 16.5 \\
\hline Bacillus thuringiensis strain BAC1141 & HM355730 & + & 15 & 21 \\
\hline Rhizobium larrymoorei strain BAC2142 & HM355731 & + & 14 & 16 \\
\hline Staphylococcus warneri strain BAC2143 & HM355732 & - & 0 & 0 \\
\hline Staphylococcus sp. strain BAC2144 & HM355733 & + & 13 & 16 \\
\hline
\end{tabular}


Table 1. Continued.

\begin{tabular}{llccc}
\hline & Number of & Halo size \\
Isolate & access & $\begin{array}{c}\text { Phytase } \\
\text { production }\end{array}$ & \multicolumn{2}{c}{$\begin{array}{c}\text { (cm h } \\
\mathbf{9 6 ~ h}\end{array}$} \\
\cline { 3 - 5 } Paenibacillus sp. strain BAC2145 & HM355734 & + & 14 & 16 \\
Bacillus bataviensis strain BAC2147 & HM355735 & - & 0 & 0 \\
Stenotrophomonas maltophilia strain BAC3148 & HM355736 & - & 0 & 0 \\
Stenotrophomonas maltophilia strain BAC3149 & HM355737 & + & 10.5 & 16.5 \\
Stenotrophomonas maltophilia strain BAC3150 & HM355738 & - & 0 & 0 \\
Bacillus thuringiensis strain BAC3151 & HM355739 & - & 0 & 0 \\
Staphylococcus warneri strain BAC1152 & HM355740 & - & 0 & 0 \\
Microbacterium testaceum strain BAC2153 & HM355741 & + & 11.5 & 18 \\
Microbacterium testaceum strain BAC3154 & HM355742 & - & 0 & 0 \\
Stenotrophomonas maltophilia strain BAC3155 & HM355743 & - & 12 & 15 \\
Delftia tsuruhatensis strain BAC1156 & HM355744 & - & 0 & 0 \\
Microbacterium foliorum strain BAC1157 & HM355745 & + & $15.0 \dagger$ & 17 \\
Delftia tsuruhatensis strain BAC2158 & HM355746 & + & 12.5 & 15 \\
Delftia tsuruhatensis strain BAC3159 & HM355747 & + & 11 & 13 \\
Stenotrophomonas maltophilia strain BAC2160 & HM355748 & + & 13 & 18 \\
Rhodococcus erythropolis strain BAC2162 & HM355749 & + & 14.5 & $22.0 \dagger$ \\
\hline
\end{tabular}

† Isolates selected for phytase activity evaluation

The phytase produced by the endophytic isolates was characterized for optimal activity temperature and $\mathrm{pH}$, substrate specificity and stability in the presence of divalent cations. $M$. foliorum BAC1157 contained the phytase with the highest optimal temperature $\left(70^{\circ} \mathrm{C}\right)$, followed by $R$. erythropolis BAC2162 and S. maltophilia BAC2135 $\left(50{ }^{\circ} \mathrm{C}\right)$ and
P. aeruginosa $\quad$ BAC3115 $\quad\left(40{ }^{\circ} \mathrm{C}\right)$

(Figure 2). The $\mathrm{pH}$ studies revealed that $M$. foliorum BAC1157, $P$. aeruginosa BAC3115 and $R$. erythropolis BAC2162 exhibit two peaks of maximum phytase activity (pH 3.0 and 5.0, 3.0 and 8.0 and 4.5 and 8.0, respectively), while $S$. maltophilia BAC2135 showed a single peak of activity at pH 4.5 (Figure 3). 


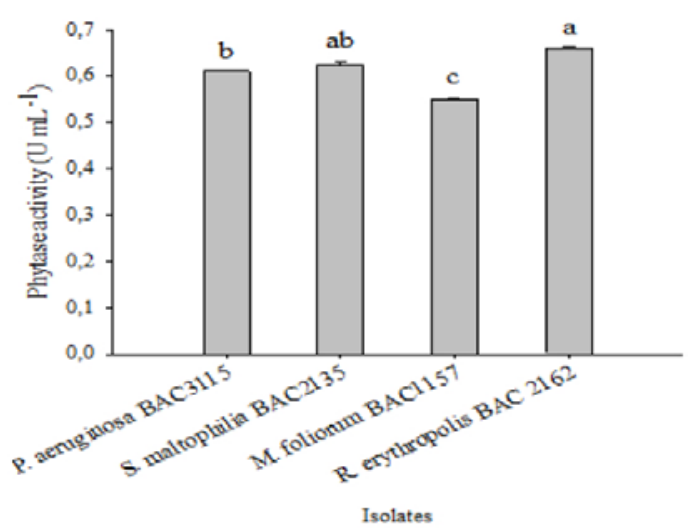

Figure1. Activity of the endophytic isolatederived phytases. The enzyme activity was measured in phytase-inducing liquid media after 48 hours of culture. 1 - Pseudomonas aeruginosa strain BAC3115; 2 Stenotrophomonas maltophilia strain BAC2135; 3 - Microbacterium foliorum strain BAC1157; 4 - Rhodococcus erythropolis strain BAC2162. The bars followed by the same letter in the same column do not differ in Tukey's test at $5 \%$ probability.

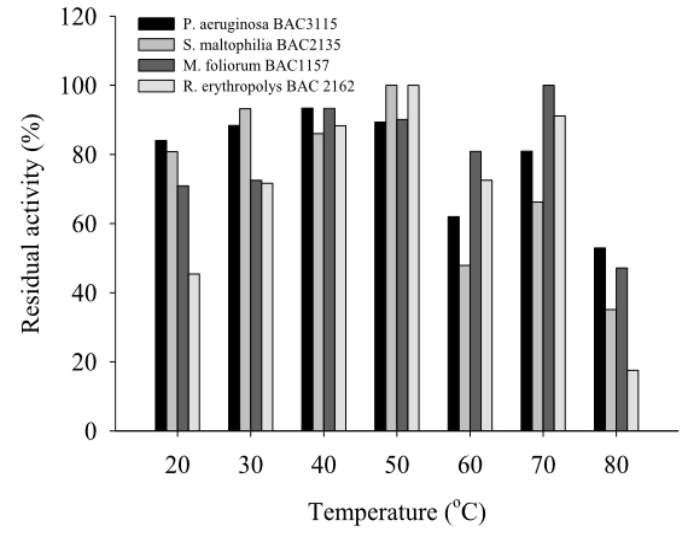

Figure 2. Optimal temperatures for the phytases derived from the four endophytic isolates. 115 - Pseudomonas aeruginosa strain BAC3115; 135 - Stenotrophomonas maltophilia strain BAC2135; $3-157$ Microbacterium foliorum strain BAC1157; 162 - Rhodococcus erythropolis strain BAC2162.The optimal temperatures were determined in phytase-inducing liquid media after 48 hours of culture.

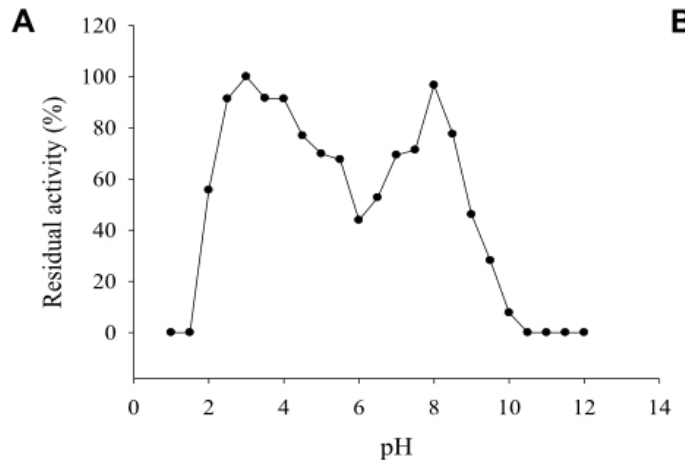

B
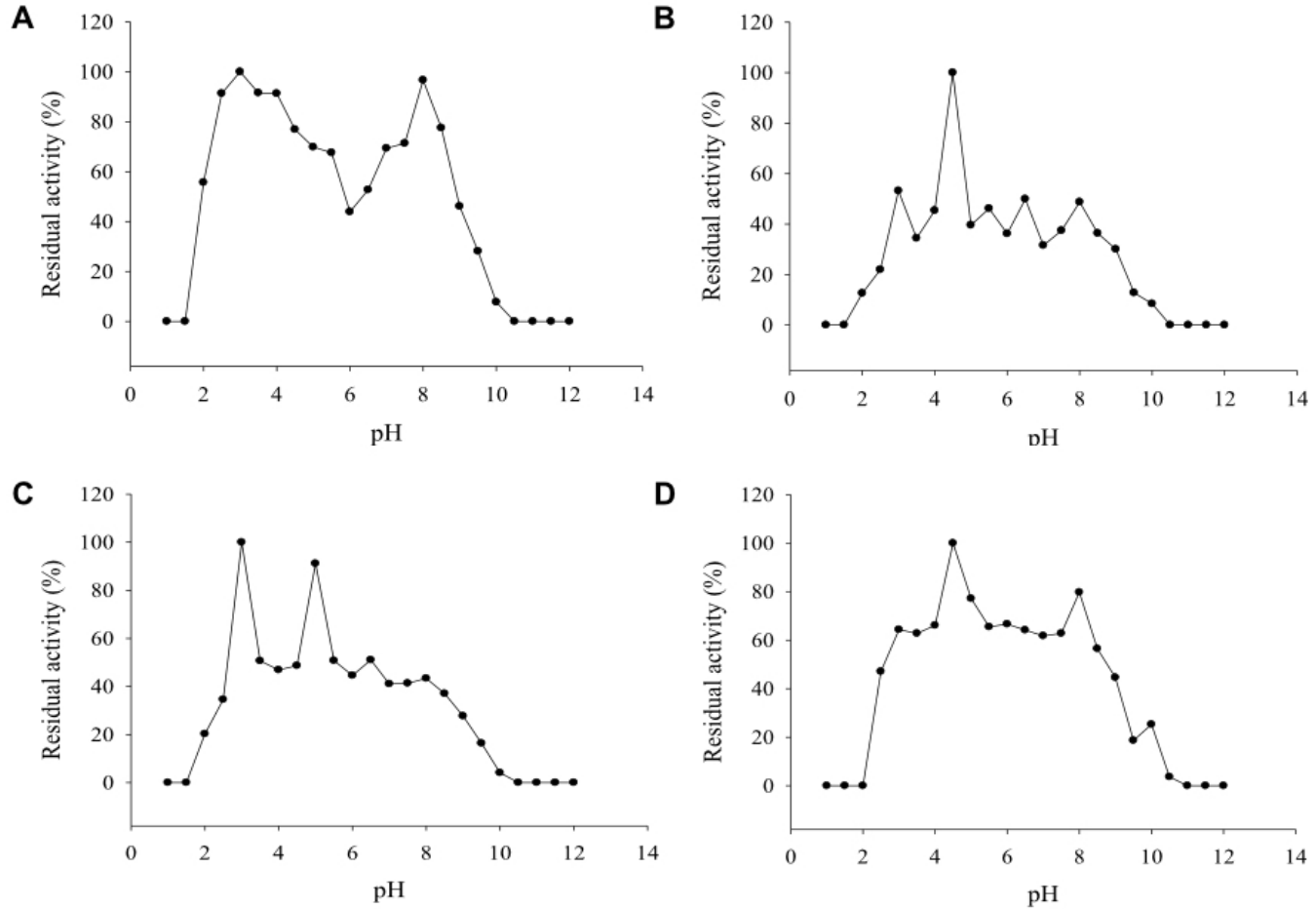

Figure 3. Optimal $\mathrm{pH}$ for the phytases derived from the four endophytic isolates. The optimal $\mathrm{pH}$ values were determined in phytase-inducing liquid media after 48 hours of culture. A - $P$. aeruginosa BAC3115, B - S. maltophilia BAC2135, C - M. foliorum BAC1157, D - R. erythropolis BAC2162. 
To determine the specific phytase activity, different phosphate sources were used to measured phytase activity from the four endophytic bacteria isolates. Activity toward $\beta$-glycerophosphate and sodium pyrophosphate was detected in the culture supernatants of the four bacterial isolates when phosphate compounds and phytic acid were both used as substrates in the reaction. S. maltophilia BAC2135 was the only bacterial isolate that showed activity with ATP, while M. foliorum BAC1157 and R. erythropolis
BAC2162 showed activity with ADP (Table 2). Bacterial phytases usually exhibit specificity for phytic acid and low activities toward other phosphorylated compounds, such as ADP, $\beta$-glycerophosphate and pyrophosphate (Shimizu, 1982; Casey and Walsh, 2003; Kim et al., 2003). As shown in Table 2, the enzymes produced by the endophytic isolates can be characterized as phytases and phosphatases with a narrow spectrum of action on other phosphorylated compounds.

Table 2. Phytase specificity for phosphorylated substrates.

\begin{tabular}{lcccc}
\hline \multirow{2}{*}{ Substrate } & \multicolumn{4}{c}{ Relative activity (\%) } \\
\cline { 2 - 5 } & BAC3115 & BAC2135 & BAC1157 & BAC2162 \\
\hline Phytate & $100 \mathrm{a}$ & $100 \mathrm{a}$ & $100 \mathrm{a}$ & $100 \mathrm{a}$ \\
B-Glycerophosphate & $35 \mathrm{c}$ & $13 \mathrm{c}$ & $20 \mathrm{~cd}$ & $59 \mathrm{~b}$ \\
ADP & $0 \mathrm{~d}$ & $0 \mathrm{c}$ & $48 \mathrm{~b}$ & $53 \mathrm{c}$ \\
ATP & $0 \mathrm{~d}$ & $15 \mathrm{c}$ & $0 \mathrm{~d}$ & $0 \mathrm{e}$ \\
Sodium pyrophosphate & $73 \mathrm{~b}$ & $60 \mathrm{~b}$ & $32 \mathrm{bc}$ & $11 \mathrm{~d}$ \\
\hline
\end{tabular}

BAC3115 - P. aeruginosa, BAC2135 - S. maltophilia, BAC1157 - M. foliorum, BAC2162 - R. erythropolis. The values followed by the same letter in the same column do not differ in Tukey's test at $5 \%$ probability.

\section{Results and discussion}

The effects of 1, 5, 10 and $20 \mathrm{mM}$ $\mathrm{Ca}^{2+}, \mathrm{Cd}^{2+}, \mathrm{Cu}^{2+}, \mathrm{Fe}^{2+}, \mathrm{K}^{+}, \mathrm{Mg}^{2+}, \mathrm{Mn}^{2+}$ and $\mathrm{Zn}^{2+}$ on the phytase activity of the four bacterial isolates can be found in Table 3 . The phytase activity of the $P$. aeruginosa BAC3115 supernatant increased with the $\mathrm{Ca}^{2+}$ concentration, and 1 and $5 \mathrm{mM} \mathrm{Cu}^{2+}$. In contrast, 10 and $20 \mathrm{mM} \mathrm{Mn}^{2+}$ and $\mathrm{Zn}^{2+}$ decreased the enzymatic activity of this isolate. Ten millimolar $\mathrm{Ca}^{2+}$ increased the phytase activity of the $S$. maltophilia BAC2135 isolate, although high $\mathrm{Mn}^{2+}$ and $\mathrm{Zn}^{2+}$ concentrations decreased the activity of this supernatant. The enzyme produced by $M$. foliorum BAC1157 showed the highest activity in high $\mathrm{Ca}^{2+}$ concentrations, 1 to $20 \mathrm{mM} \mathrm{Fe}^{2+}$, high $\mathrm{Mg}^{2+}$ and low $\mathrm{Mn}^{2+}$ and $\mathrm{Zn}^{2+}$ concentrations. The phytase produced by $R$. erythropolis BAC2162 was the most active in high $\mathrm{Fe}^{2+}$ concentrations and low $\mathrm{Mn}^{2+}$ concentrations. 
Table 3. Effect of divalent cations on the activity of endophytic isolate-derived phytases.

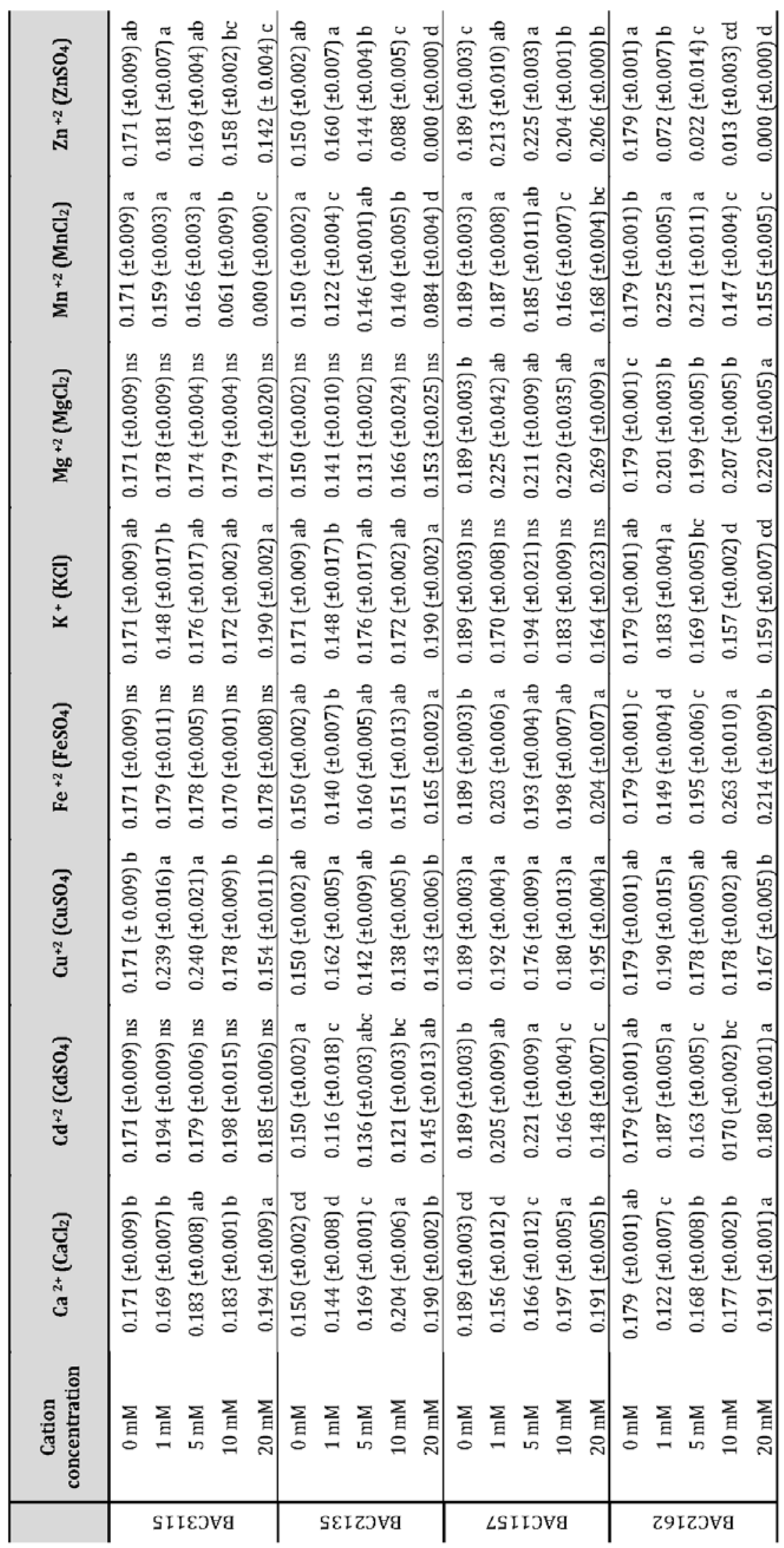


The large number of phytaseproducing bacterial isolates found in this work suggests that endophytic bacteria are promising candidates for the production and study of this enzyme. While assessing the potential production of bacterial phytase in Malaysia, Hussin et al. (2009) reported that endophytic bacteria exhibited the highest phytase activity. Endophytic microorganisms can be vertically transmitted to seeds. Considering that phytic acid is the main storage form of $\mathrm{Pi}$ in grains and legumes, it is plausible that a selection of endophytic microorganisms capable of degrading phytic acid and assisting in the process of seed germination might exist (López-López et al., 2010).

Extracellular enzymes are excellent candidates for large-scale production and marketing because they eliminate the expenses associated with the extraction process (Anis Shobirin et al., 2009). The most production of bacterial phytases are intracellular, with the exception of those phytases that are derived from Bacillus subtilis, Lactobacillus amylovorus and Enterobacter sp. 4 species (Vohra and Satyanarayana, 2003). However, in the current study, those bacterial isolates presenting the largest hydrolysis halos around the colonies in solid media containing calcium phytate suggests an extracellular phytase activity. Anis Shobirin et al. (2009) also described two bacterial isolates of the Pantoea stewartii species as extracellular phytase producing bacteria. Consequently, the Microbacterium, Pseudomonas, Rhodococcus and Stenotrophomonas genera should be included among those genera with extracellular phytase activity.

To assess the potential application of the phytases produced by $P$. aeruginosa BAC3115, S. maltophilia BAC2135, M. foliorum BAC1157 and $R$. erythropolis BAC2162 in the animal feed industry, the enzymes produced by these bacteria were characterized for optimal activity $\mathrm{pH}$ and temperature, substrate specificity and divalent cations effect. The phytase produced by the $M$. foliorum BAC1157 isolate was the most active at $70{ }^{\circ} \mathrm{C}$. Furthermore, the phytase in question showed $90 \%$ activity at $40{ }^{\circ} \mathrm{C}$, which is another desirable feature, as the body temperature of pigs is $39{ }^{\circ} \mathrm{C}$ (Casey and Walsh, 2003). The optimal temperature for most microbial phytases is approximately $50{ }^{\circ} \mathrm{C}$ (Simon and Igbasan, 2002); however, some microbial phytases have exhibited optimal temperatures above $60{ }^{\circ} \mathrm{C}$ (Berka et al., 1998; Matsui et al., 2000; Zamudio et al., 2001). M. foliorum BAC1157 isolate has a remarkable application and can be used as a supplement in swine feed because the pelleting process involves temperatures above $70^{\circ} \mathrm{C}$.

The endophytic isolates used in the here exhibited more than one optimal pH peak, suggesting the production of phytases with different characteristics. B. subtilis (natto) produces a phytase with an optimal activity between 6.0 and 6.5 (Shimizu, 1992), and the $C$. braakii phytase shows optimal activity at a $\mathrm{pH}$ of approximately 4.0 (Kim et al., 1998). The phytase produced by $M$. foliorum BAC1157 showed the highest enzymatic activity at a pH of 3.0 and merely $20 \%$ activity at $\mathrm{pH}$ 2.0. The $\mathrm{pH}$ in the swine stomach varies between 2.0 and 4.0 (Lindberg and Ogel, 2001) depending on the feed and other factors. This substantial loss of activity at $\mathrm{pH} \quad 2.0$ is a negative factor, but controlling animal feed can ensure that the stomach $\mathrm{pH}$ does not reach this value. However, the low $\mathrm{pH}$ in gastric phase promotes the environment where phytate is really soluble and most susceptible to hydrolyses. Although for some fungal phytases it seems to be a huge problem because of the range of optimal activity at high $\mathrm{pH}$. On the other hand, isolating and characterizing the gene encoding the M. foliorum BAC1157 phytase may aid in the development of site-directed mutagenesis strategies designed to increase enzymatic activity at $\mathrm{pH}$ values below 4.0, similar to the 
phytase applied in the animal feed industry, as the produced by Aspergillus niger NRRL3135 (Mullaney et al., 2002).

Because the several divalent cations found in the diets administered to monogastric animals may interfere with the activity of supplemental enzymes added to these foods, it is necessary to predict the effect of such cations on phytase activity. The addition of cations to the enzymatic reaction changed the phytase activity of the four bacterial isolates. The $M$. foliorum BAC1157 bacterial isolate was only inhibited at high concentrations of $\mathrm{Cd}^{2+}$ and $\mathrm{Mn}^{2+}$, in addition to showing phytase activity at $70{ }^{\circ} \mathrm{C}$. Furthermore, increased phytase activity in the presence of $\mathrm{Zn}^{2+}$ is an uncommon feature, especially considering that this metal inhibits several of the phytases reported in the literature (Yoon et al., 1996; Greiner et al., 1997; Greiner et al., 2009). Studies have reported that the presence of $\mathrm{Ca}^{2+}$ did not affect the activity of $A$. niger 11T53A9 (Greiner et al., 2009) and $C$. braaki phytases (Kim et al., 1998). However, $\mathrm{Ca}^{2+}$ inhibited the activity of a phytase produced by A. niger ATCC 9142 (Casey and Walsh, 2003), and decreased the activity of a phytase produced by $B$. subtilis DS11 (Kim et al., 1998). Similarly, $\mathrm{Fe}^{2+}$ and $\mathrm{Fe}^{+3}$ did not affect the activity of the phytase produced by $A$. niger ATC9142 (Casey and Walsh, 2003), despite inhibiting some other phytases (Kim et al., 1998; Greiner et al., 2009). Increased activity in the presence of cations such as $\mathrm{Cu}^{2+}, \mathrm{Mg}^{2+}, \mathrm{Mn}^{2+}$ and $\mathrm{Zn}^{2+}$ was another unusual feature noted in the phytase isolated by Casey and Walsh (Casey and Walsh, 2003).

\section{Conclusion}

The results of the current study suggest that $P$. aeruginosa BAC3115, $R$. erythropolis BAC2162, S. maltophilia BAC2135 and M. foliorum BAC1157 are promising candidates for the study and application of endophytic isolate-derived phytases in the feed industry. To our knowledge, this is the first report on phytase production by the bacteria Microbacterium foliorum BAC1157, in which this phytase could has a potential use in monogastric diets.

\section{Acknowledgments}

We thank the Brazilian institutions Coordenação de Aperfeiçoamento de Pessoal de Nível Superior (CAPES), Conselho Nacional de Desenvolvimento Científico e Tecnológico (CNPq) and Fundação de Amparo à Pesquisa do Estado de Minas Gerais (FAPEMIG) for financial support.

\section{Conflict of interest}

The authors declare that they have no conflict of interest.

\section{Referências}

Anis Shobirin, M. H.; Farouk, A.; Greiner, R. Potential phytate-degrading enzyme producing bacteria isolated from Malaysian maize plantation. African Journal of Biotechnology, v. 8 , no. 15 , p. $3540-3546$, 2009.

Araujo, W. L.; Marcon, J.; Maccheroni, W., Jr; Van Elsas, J. D.; Van Vuurde, J. W.; Azevedo, J. L. Diversity of endophytic bacterial populations and their interaction with Xylella fastidiosa in citrus plants. Applied and Environmental Microbiology, v. 68, p. 4906-4914, 2002. https://doi.org/ 10.1128/AEM.68.10.4906-4914.2002

Ariza, A.; Moroz, O. V.; Blagova, E. V.; Turkenburg, J. P.; Waterman, J.; Roberts, S. M.; Vind, J.; Sjøholm, C.; Lassen, S. F.; Maria, L.; Glitsoe, V.; Skov, L. K.; Wilson, K. S. Degradation of phytate by the 6-phytase from Hafnia alvei: A combined structural and solution study. PLoS ONE, 8:5, e65062, 2013. https://doi.org/10.1371/journal.pone.00650 62

Berka, R. M.; Rey, M. W.; Brown, K. M.; Byun, T.; Klotz, A. V. Molecular characterization and expression of a phytase gene from the thermophilic fungus Thermomyces lanuginosus. Applied and Environmental Microbiology, v. 64, p. 4423-4427, 1998. Available

from: 
<https://aem.asm.org/content/64/11/4423. long>. Accessed on: Sept. 23, 2018.

Carrington, A. L.; Calcutt, N. A.; Ettlinger, C. B.; Gustafsson, T.; Tomlinson, D. R. Effects of treatment with myo-inositol or its 1,2,6trisphosphate (PP56) on nerve conduction in streptozotocindiabetis. European Journal of Pharmacology, v. 237 , no. $2 / 3$, p. 257-263, 1993. https://doi.org/10.1016/00142999(93) 90277-0

Casey, A.; Walsh G. Purification and characterization of extracellular phytase from Aspergillus niger ATCC 9142. Bioresource Technology, v. 86, p. 183-188, $2003 . \quad$ https://doi.org/10.1016/S09608524(02)00145-1

Claxson, A.; Morris, C.; Blake, D.; Sirén, M.; Halliwell, B.; Gustafsson, T.; Löfkvist, B.; Bergelin, I. The anti-inflammatory effects of D-myo-inositol-1.2,6-trisphosphate (PP56) on animal models of inflammation. Agents and Actions, v. 29, p. 68-70, 1990.

Coelho, C. M. M.; Santos, J. C. P.; Tsai, S. M.; Vitorello, V. A. Seed phytate content and phosphorus uptake and distribution in dry bean genotypes. Brazilian Journal of Plant Physiology, v. 14, no. 1, p. 51-58, 2002. https://doi.org/10.1590/S1677-0420 2002000100007

Corrêa, T. L. R.; Queiroz, M. V.; Araújo, E. F. Cloning, recombinant expression and characterization of a new phytase from Penicillium chrysogenum. Microbiological Research, v. 170, p. 205-212, 2015. https://doi.org/10.1016/j.micres.2014.06.00 5

Costa, L. E. O.; Queiroz, M. V.; Borges, A. C.; Moraes, C. A.; Araújo, E. F. Isolation and characterization of endophytic bacteria isolated from the leaves of the common bean (Phaseolus vulgaris). Brazilian Journal of Microbiology, v. 43, no. 4, p. 1562-1575, 2012. https://doi.org/10.1590/S1517-8382 2012000400041

Greiner, R.; Haller, E.; Konietzny, U.; Jany, K.D. Purification and characterization of a phytase from Klebsiella terrigena. Archives of Biochemistry and Biophysics, v. 341, no. 2, p. 201-206, 1997. https://doi.org/ 10.1006/abbi.1997.9942

Greiner, R.; Silva, L. G.; Couri S. Purification and characterisation of an extracellular phytase from Aspergillus niger 11T53A9. Brazilian Journal of Microbiology, v. 40, p. 795-807, 2009. https://doi.org/10.1590/ S1517-83822009000400010

Hallmann, J.; Quadt-Hallmann, A.; Mahaffee, W. F.; Kloepper, J. W. Bacterial endophytes in agricultural crops. Canadian Journal of Microbiology, v. 43, p. 895-914, 1997. https://doi.org/10.1139/m97-131

Heinonen, J. K.; Lahti, R. J. A new and convenient colorimetric determination of inorganic orthophosphate and its application to the assay of inorganic pyrophosphatase. Analytical Biochemistry, v. 113, no. 2, p. 313-317, 1981. https://doi.org/10.1016/ 0003-2697(81)90082-8

Hussin, A.S. M.; Farouk, A.; Greiner, R. Potencial phytate-degrading enzyme producing bacteria isolated from Malaysian maize plantation. African Journal of Biotechnology, v. 8, p. 3540-3546, 2009.

Kiarie, E.; Romero, L. F.; Nyachoti, C. M. The role of added feed enzymes in promoting gut health in swine and poultry. Nutrition Research Review, v. 26, no. 1, p. 71-88, 2013. https://doi.org/10.1017/S09544224 13000048

Kim, H.; Kim, Y.; Lee, J.; Kim, K.; Kim, Y. Isolation and characterization of a phytase with improved properties from Citrobacter braakii. Biotechnology Letters, v. 25, no. 15, p. 1231-1234, 2003. https://doi.org/ 10.1023/A:1025020309596

Kim, Y.; Kim, H.; Bae, K.; Yu, J.; Oh, T. Purification and properties of a thermostable phytase from Bacillus sp. DS11. Enzyme and Microbial Technology, v. 22, no. 1, p. 2-7, 1998. https://doi.org/10.1016/S0141-0229 (97)00096-3

Konietzny, U.; Greiner, R. Molecular and catalytic properties of phytate-degrading enzymes (phytases). International Journal of Food Science +Technology, v. 37, p. 791812, 2002. https://doi.org/10.1046/ j.13652621.2002.00617.x

Lei, X. G.; Porres, J. M. Phytase enzymology, applications, and biotechnology. Biotechnology Letters, v. 25, no. 21, p. 1787-1794, 2003. https://doi.org/ 10.1023/A:1026224101580

Lindberg, J. E.; Ogel, B. Digestive physiology of pigs. Oxford, UK: CAB International, 2001.

López-López, A.; Rogel, M. A.; Ormeño-Orrillo, E.; Martínez-Romero, J.; Martínez-Romero, E. Phaseolus vulgaris seed-borne endophytic community with novel bacterial species such 
as Rhizobium endophyticum sp. nov. Systematic and Applied Microbiology, v. 33, p. 322-327, 2010. https://doi.org/ 10.1016/j.syapm.2010.07.005

Maffucci, T.; Piccolo, E.; Cumashi, A.; Iezzi, M.; Riley, A. M.; Saiardi, A.; Godage, H. Y.; Rossi, C.; Broggini, M.; Iacobelli, S.; Potter, B. V. L.; Innocenti, P.; Falasca, M. Inhibition of the phosphatidylinositol 3-Kinase/Akt pathway by inositol pentakisphosphate results in antiangiogenic and antitumor effects. Cancer Research, v. 65, no. 18, p. 8339-8349, 2005. https://doi.org/10.1158/0008-5472.CAN05-0121

Matsui, T.; Nakagawa, Y.; Tamura, A.; Watanabe, C.; Fujita, K.; Nakajima, T.; Yano, H. Efficacy of yeast phytase in improving phosphorus bioavailability in a corn-soybean meal-based diet for growing pigs. Journal of Animal Science, v. 78, p. 94-99, 2000. https://doi.org/10.2527/2000.78194x

Mullaney, E. J.; Daly, C. B.; Kim, T.; Porres, J. M.; Lei, X. G.; Sethumadhavan, K.; Ullah, A. H. J. Site-directed mutagenesis of Aspergillus niger NRRL 3135 phytase at residue 300 to enhance catalysis at $\mathrm{pH}$ 4.0. Biochemical and Biophysical Research Communications, v. 297, p. 1016-1020, 2002. https://doi.org/10.1016/S0006-291X(02) 02325-2

Mullaney, E. J.; Ullah, A. H. The term phytase comprises several different classes of enzymes. Biochemical and Biophysical Research Communications, v. 312, no. 1, p. 179-184, 2003. https://doi.org/10.1016/ j.bbrc.2003.09.176

Patki, J. M.; Singh, S.; Mehta, S. Partial purification and characterization of phytase from bacteria inhabiting the mangroves of the Western Coast of India. International Journal of Current Microbiology and Applied Sciences, v. 4, no. 9, p. 156-169, 2015. Available from: <https://www.ijcmas. com/vol-4-9/J.M. Patki, et al.pdf $>$. Accessed on: Sept. 23, 2018.

Rao, D. E.; Rao, K. V.; Reddy, T. P.; Reddy, V. D. Molecular characterization, physicochemical properties, known and potential applications of phytases: An overview. Critical Reviews in Biotechnology, v. 29, no. 2, p. 182-198, 2009. https://doi.org/10.1080/07388550 902919571

Schroder, B.; Breves, G.; Rodehutscord, M. Mechanisms of intestinal phosphorus absorption and availability of dietary phosphorus in pigs. Deutsche tierärztliche Wochenschrift, v. 103, p. 209-214, 1996.

Shimizu, M. Purification and characterization of phytase from Bacillus subtilis (natto) N-77.

Bioscience, Biotechnology, and Biochemistry, v. 56, p. 1266-1269, 1992. https://doi.org/10.1271/bbb.56.1266

Simon, 0.; Igbasan, F. In vitro properties of phytases from various microbial origins. International Journal of Food Science +Technology, v. 37, p.813-822, 2002. https://doi.org/10.1046/j.1365-2621.2002. 00621.x

Strobel, G. A.; Daisy, B. Bioprospecting for microbial endophytes and their natural products. Microbiology and Molecular Biology Reviews, v. 67, p. 491-502, 2003. https://doi.org/10.1128/MMBR.67.4.491502.2003

Tang, J.; Leung, A.; Leung, C.; Lim, B. L. Hydrolysis of precipitated phytate by three distinct families of phytases. Soil Biology and Biochemistry, v. 38, p. 1316-1324, 2006. https://doi.org/10.1016/j.soilbio. 2005.08.021

Tortola, L.; Souza, N. G.; Zaine, L.; Gomes, M. O.; Matheus, L. F.; Vasconcellos, R. S.; Pereira, G. T.; Carciofi, A. C. Enzyme effects on extruded diets for dogs with soybean meal as a substitute for poultry by-product meal. Journal of Animal Physiology and Animal Nutrition, v. 97, no. 1, p.39-50, 2013. https://doi.org/10.1111/jpn.12009

Turner, B. L.; Haygarth, P. M. Phosphorus forms and concentrations in leachate under four grassland soil types. Soil Science Society of America Journal, v. 64, no. 3, p. 1090-1099, 2000. https://doi.org/ 10.2136/sssaj2000.6431090x

Urbano, G.; López-Jurado, M.; Aranda, P.; Vidal-Valverde, C.; Tenorio, E.; Porres, J. The role of phytic acid in legumes: Antinutrient or beneficial function? Journal of Physiology and Biochemistry, v. 56, p. 283-294, 2000. https://doi.org/10.1007/BF03179796

Vohra, A.; Satyanarayana, T. Phytases: Microbial sources, production, purification, and potential biotechnological applications. Critical Reviews in Biotechnology, v. 23, no. 1 , p. $29-60$, 2003. https://doi.org/ $10.1080 / 713609297$

Yoon, S. J.; Choi, Y. J.; Min, H. K.; Cho, K. K.; Kim, J. W.; Lee, S. C.; Jung, Y. H. Isolation and identification of phytase-producing 
bacterium, Enterobacter sp. 4, and enzymatic properties of phytase enzyme. Enzyme and Microbial Technology, v. 18, p. 449-454, 1996. https://doi.org/10.1016/0141-0229 (95)00131-X

Zamudio, M.; González, A.; Medina, J. A. Lactobacillus plantarum phytase activity is due to non-specific acid phosphatase. Letters in Applied Microbiology, v. 32, p. 181-184, 2001. https://doi.org/10.1046/j.1472-765x. 2001.00890.x

License information: This is an open-access article distributed under the terms of the Creative Commons
(CC) Attribution License, which permits unrestricted use, distribution, and reproduction in any medium, provided the original work is properly cited. 